\title{
Electron transport through silicon multiple quantum dot array devices
}

\author{
Gento Yamahata $^{1^{*}}$, Yoshishige Tsuchiya ${ }^{1,2,4}$, Hiroshi Mizuta ${ }^{2,3,4}$, and Shunri Oda ${ }^{1,4}$ \\ ${ }^{1}$ Quantum Nanoelectronics Research Center, Tokyo Institute of Technology, \\ 2-12-1, O-okayama, Meguro-ku, Tokyo 152-8552, Japan. \\ ${ }^{2}$ School of Electronics and Computer Science, University of Southampton, Southampton, U.K. \\ ${ }^{3}$ Department of Physical Electronics, Tokyo Institute of Technology, Tokyo, Japan. ${ }^{4}$ SORST-JST. \\ *Phone: +81-3-5734-3854 FAX: +81-3-5734-2542 E-mail: ygent@neo.pe.titech.ac.jp
}

\section{Introduction}

In recent years, silicon double quantum dot $(\mathrm{Si}$ DQD) structures have been attracting much attention as a building block for the quantum information devices (QIDs). To realize the QIDs, coherent manipulation of two qubits is required as well as that of one qubit. Hence, it is crucial to analyze the interaction between two DQDs [1]. In addition, the two DQDs system is applicable for the quantum cellular automaton [2]. So far, we have investigated the electrostatic coupling in Si DQDs [3]. In this paper, we study the electron transport through the Si multiple quantum dot array devices (Si MQDADs), and observe the strong correlation between two DQDs.

\section{Structure and Fabrication of the Si MQDADs}

Figure 1(a) shows the schematic image of the $\mathrm{Si}$ MQDADs. Since we need strong electrostatic couplings between two DQDs, DQD1 and DQD2 are physically connected each other. The MQDADs were defined on the silicon-on-insulator (SOI) of about $40 \mathrm{~nm}$ and the buried oxide of $200 \mathrm{~nm}$ in thickness. First, a 40-nm-thick SOI film, whose thickness was reduced via thermal oxidation, was doped heavily by ion implantation (n-type, phosphorous). The MQDADs were then patterned using the electron beam lithography. The electron cyclotron resonance reactive ion etching was used to transfer the resist pattern onto the SOI layer. Thermal oxidation was then done for $30 \mathrm{~min}$ at $1000{ }^{\circ} \mathrm{C}$ in order to passivate the surface states and reduce the dot size. Finally, Ohmic contacts were formed by evaporating about 300-nm-thick Al. Figure 1(b) shows the scanning electron micrograph (SEM) image of the MQDADs.

\section{Measurement Results and Discussion}

All measurements were performed for the MQDADs using the Hewlett Packard 4156A parameter analyzer at the temperature of $4.2 \mathrm{~K}$ in liquid helium. Figures 2(a)-2(d) show the currents $I_{T 1}, I_{T 2}, I_{T 3}$, and $I_{T 4}$ measured at the terminals T1, $\mathrm{T} 2, \mathrm{~T} 3$, and $\mathrm{T} 4$, respectively, as a function of the voltage of $\mathrm{T} 1\left(V_{T 1}\right)$, where the terminals of $\mathrm{T} 2, \mathrm{~T} 3$, and T4 are grounded. As shown in the insets of Fig. 2(a) and 2(c), the currents flow from the terminal $\mathrm{T} 1$ to the terminal $\mathrm{T} 3$ in the low bias region $(-20$ $\left.\mathrm{mV}<V_{T 1}<10 \mathrm{mV}\right)$. On the other hand, in the high bias region $\left(10 \mathrm{mV}<V_{T 1},-20 \mathrm{mV}>V_{T 1}\right), I_{T 2}$ and $I_{T 4}$ begin to flow in the direction from DQD2 to DQD1, indicating Coulomb blockade between DQD1 and DQD2 is lifted. Note that $I_{T 3}$ is very low probably because of the weak coupling of the right part between two DQDs.

Figures 3(a)-3(d) show the contour plots of the $I_{T 1}-I_{T 4}$, respectively, as a function of the voltage of G1 and G4 $\left(V_{G 1}, V_{G 4}\right)$, where $V_{T 1}$ is $-6 \mathrm{mV}$ and the other terminals are grounded. We observed the two current paths; one is in the direction from DQD2 to DQD1 (Path A) [see the white circle in Fig. 3(a)], the other is through the individual DQD (Path B) [see the white circle in Fig. 3(c)]. In addition, the current peaks through the path A appear at the top right corner, which are blown up in the inset of the Fig. 3(a), implying the periodic current oscillations. To focus on the current through the path A, Figs. 4(a)-4(d) show the contour plots of the currents $I_{T 1}-I_{T 4}$, respectively, as a function of $V_{G 1}$ and $V_{G 4}$, where the voltage of the terminals $\mathrm{T} 1, \mathrm{~T} 2$, and $\mathrm{T} 3$ are $-6 \mathrm{mV}$ and the terminal of $\mathrm{T} 4$ is grounded. $I_{T 1}$ and $I_{T 3}$, corresponding the currents through the path A, show the complicated behavior because of the electrostatic interaction in the two DQDs. On the other hand, $I_{T 2}$ and $I_{T 4}$ include the current through not only the path A but also the path B. The latter periodic current oscillations show the single dot property because of the strong coupling in DQD2. Note that while the maximal $I_{T I}$ is about $-1 \mathrm{pA}$, the local shift of the current $I_{T 2}$, as shown in the white circle in Fig. 4(b), is one order of magnitude higher than that of $I_{T 1}$. This result indicates the electron configuration in the DQD1 strongly influences on the current through DQD2, and vice versa.

\section{Summary}

We fabricated the Si MQDADs and measured their electron transport properties. The currents through the MQDADs were analyzed and the strong interaction between the two DQDs was observed. The MQDADs are promising candidates 
for the novel information devices.

\section{Acknowledgment}

One of authors, (G. Y.), is supported by the JSPS Research Fellowship for Young Scientists.

\section{References}

[1] G. Shinkai, et al., Appl. Phys. Lett. 90, 103116 (2007).

[2] M. Mitic, et al., Appl. Phys. Lett. 89, 013503 (2006).

[3] G. Yamahata, et al., SSDM, p.598 (2007). (a)

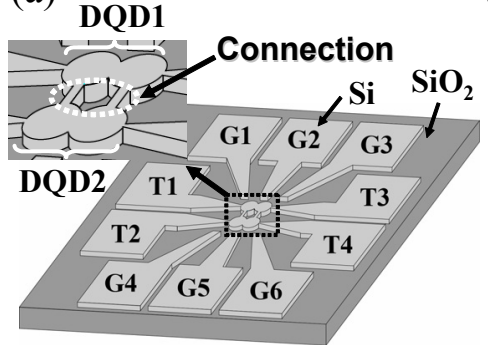

(b)

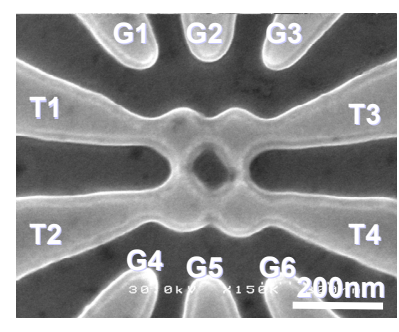

Fig. 1 (a) Schematic and (b) SEM images of the MQDADs

(a)

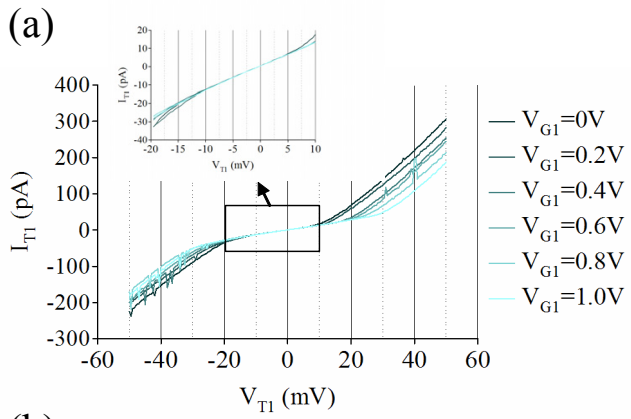

(b)

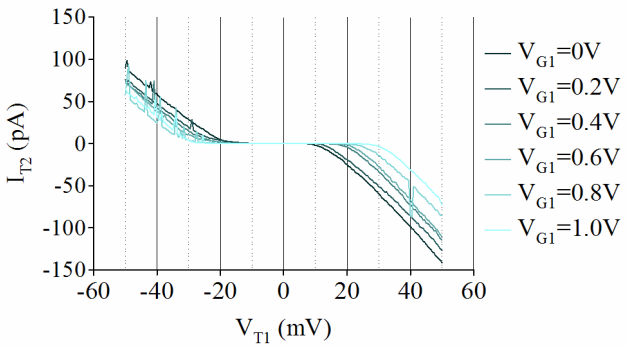

(c)

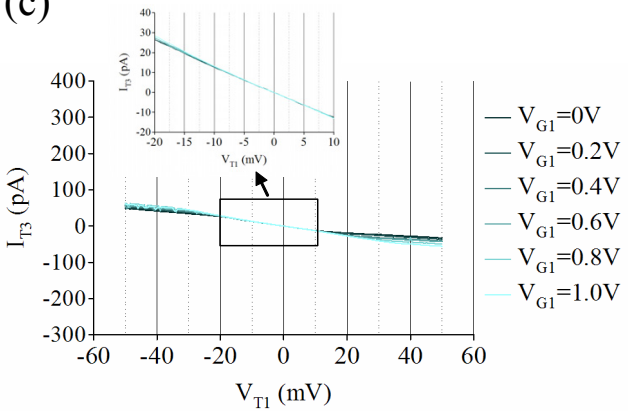

(d)

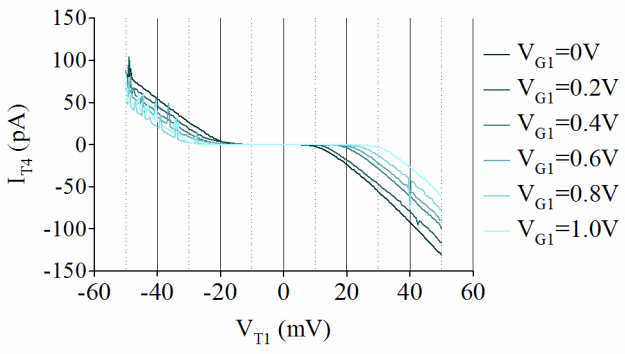

Fig. 2 (a)-(d) $I_{T 1}, I_{T 2}, I_{T 3}$, and $I_{T 4}$ versus $V_{T 1}$ characteristics with different gate voltages $V_{g l}$. (a)

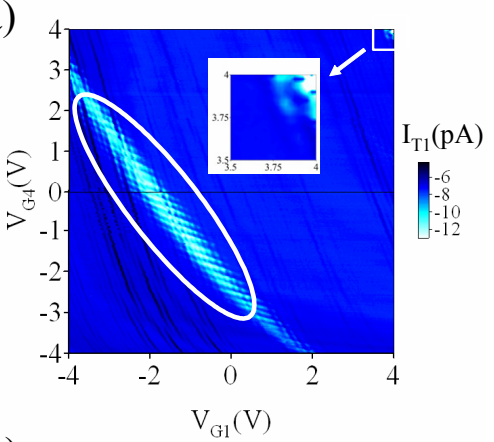

(b)

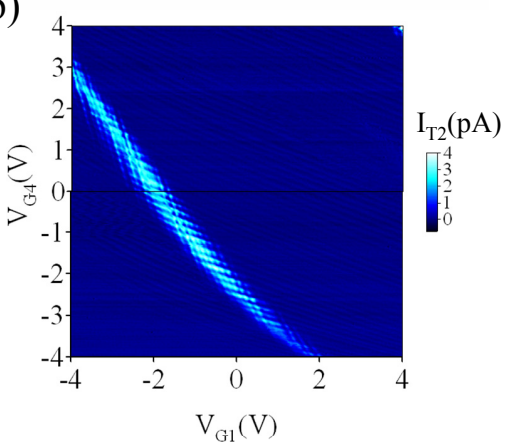

(c)

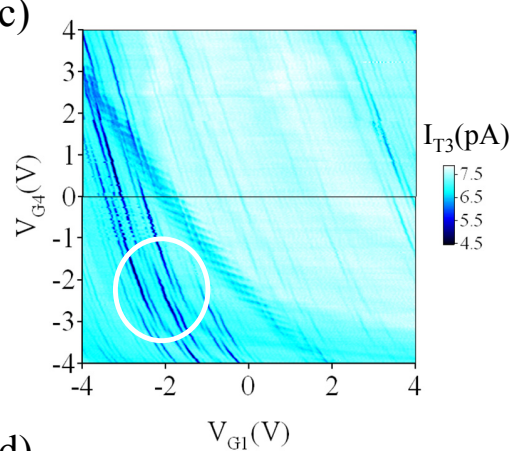

(d)

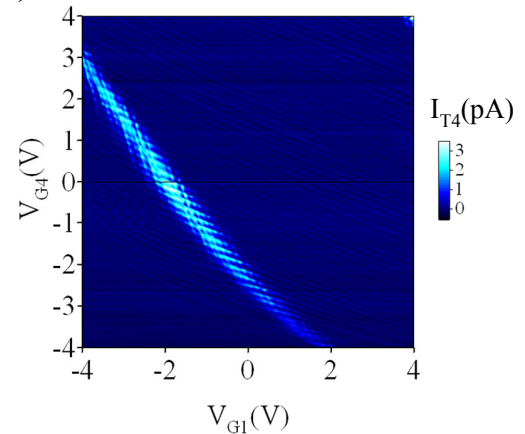

Fig. 3 (a)-(d) the contour plots of $I_{T 1}$, $I_{T 2}, I_{T 3}$, and $I_{T 4}$ versus $V_{G 1}, V_{G 4}$, where $V_{T 1}=-6 \mathrm{mV}$ and $V_{T 2}, V_{T 3}$, and $V_{T 4}$, are grounded.

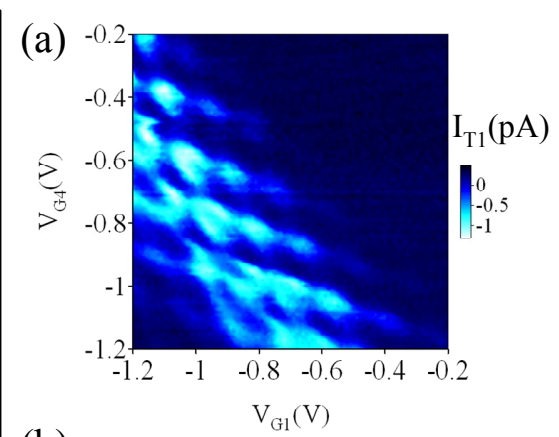

(b)

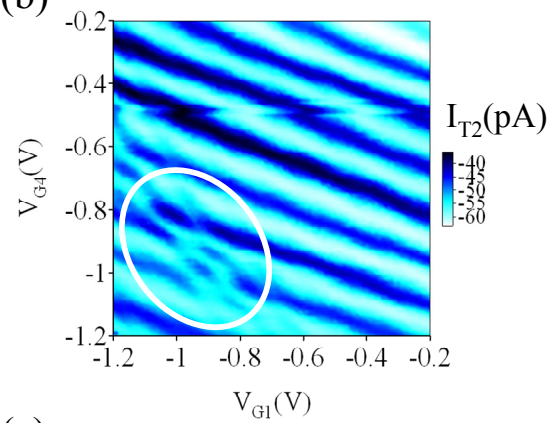

(c)

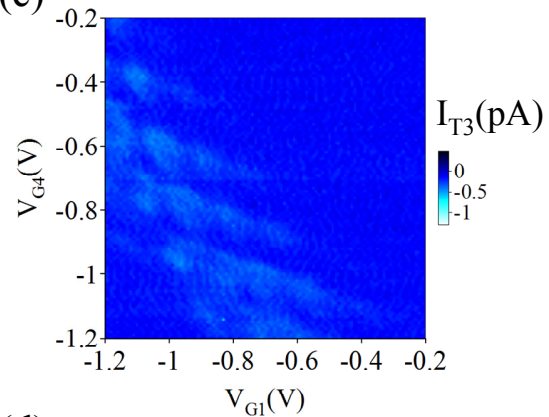

(d)

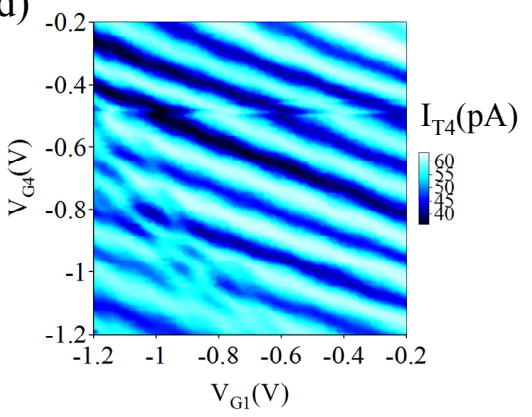

Fig. 4 (a)-(d) the contour plots of $I_{T 1}, I_{T 2}, I_{T 3}$, and $I_{T 4}$ versus $V_{G 1}, V_{G 4}$ where $V_{T 1}$, $V_{T 2}$, and $V_{T 3}$ are $-6 \mathrm{mV}$ and $V_{T 4}$ is grounded. 